\title{
33. Mass Distributions on the Ideal Boundaries
}

\author{
By Zenjiro KuRAMOCHI \\ Mathematical Institute, Hokkaido University \\ (Comm. by K. KunUGi, M.J.A., March 12, 1960)
}

In the previous paper ${ }^{1)}$ our proof of 3 ) of Theorem 8 is incomplete. The purpose of the present paper is to introduce a new operation, to give a proof of the above theorem and to consider mass distributions.

Let $U(z)$ be a positive harmonic function in $R-R_{0}$ such that $U(z)=0$ on $\partial R_{0}$ and $D(\min (M, U(z))<\infty$ for $M<\infty$. Let $D$ be a compact or non-compact domain. Let $U_{D_{n}}(z)$ be a harmonic function in $R-R_{0}-\left(D \cap R_{n}\right)$ such that $U_{D_{n}}(z)=0$ on $\partial R_{0}, U_{D_{n}}(z)=U(z)$ on $D \cap R_{n}$ and $U_{D_{n}}(z)$ has the minimal Dirichlet integral (M.D.I.) over $R-R_{0}$ $-\left(D \cap R_{n}\right)$. Put $U_{D}(z)=\lim _{n} U_{D_{n}}(z)$. If $U(z) \geqq U_{D}(z)$ for every domain $D$, we call $U(z)$ a superharmonic function in $\bar{R}$.

In the sequel, we use the same notations and terminologies as in the previous paper. Let $U(z)$ be a positive harmonic function in $R-R_{0}$ and superharmonic in $\bar{R}$. Then $\max _{z \in R_{n}} U(z)<M$, whence $D(\min (U(z), M))$ $<\infty$. Let $U_{n}(z)$ be a harmonic function in $R_{n}-R_{0}$ such that $U_{n}(z)=U(z)$ in $R_{n}-R_{0}$, and $U_{n}(z)$ has M.D.I. over $R-R_{n}$. Then

$$
U(z)=\lim _{n} U_{n}(z) \text {. }
$$

Operation. ${ }_{D}[U(z)]^{*}$. Let $D$ be a non-compact domain in $R$. Let ${ }_{D_{n}}[U(z)]^{*}$ be a continuous function in $R$ such that $U_{n}(z)-{ }_{D_{n}}[U(z)]^{*}$ is harmonic in $\bar{D} \cap \bar{R}_{n}, D_{n}[U(z)]^{*}$ is harmonic in $R-R_{0}-\left(\bar{D} \cap \bar{R}_{n}\right)$, ${ }_{D_{n}}[U(z)]^{*}=0$ on $\partial R_{0}$ and ${ }_{D_{n}}[U(z)]^{*}$ has M.D.I. over $R-R_{0}-\left(\bar{D} \cap \bar{R}_{n}\right)$. Then since $\bar{D}-\bar{R}_{n}$ is compact, ${ }_{D_{n}}[U(z)]^{*}$ is uniquely determined. In fact, since $U_{n}(z)$ is superharmonic in $\bar{R}$ and $U_{n}(z)$ has M.D.I. over $R-R_{n}, U_{n}(z)$ is representable by a unique mass distribution on $\partial R_{n}$ such that $U_{n}(z)=\int_{\partial R_{n}} N(z, p) d \mu_{n}(p)$. Let ${ }_{1} \mu_{n}(p)$ be the restriction of $\mu_{n}(p)$ on $\bar{D} \cap \partial R_{n}$. Then

$$
{ }_{D_{n}}[U(z)]^{*}=\int N(z, p) d_{1} \mu_{n}(p) .
$$

Now ${ }_{2} \mu_{n}(p)=\mu_{n}(p)-{ }_{1} \mu_{n}(p)$ is also a positive mass distribution, which implies that $U_{n}(z)-{ }_{D_{n}}[U(z)]^{*}$ is superharmonic $\bar{R}$. Let $\left\{n^{\prime}\right\}$ be a subsequence of $\{n\}$ such that ${ }_{D_{n}}[U(z)]^{*}$ converges uniformly in $R$. Put

1) Z. Kuramochi: Mass distributions on the ideal boundary of Riemann surfaces, II, Osaka Math. Jour., 8 (1956). 
${ }_{D}[U(z)]^{*}=\lim _{n^{\prime}}{ }_{D_{n}}[U(z)]^{*} . \quad{ }_{D}[U(z)]^{*}$ depends on $D$ and the subsequence $\left\{n^{\prime}\right\}$.

Theorem. Let $D_{1}$ and $D_{2}$ be two non-compact domains and $\left\{n^{\prime}\right\}$ be a subsequence such that ${ }_{1} D_{n}[U(z)]^{*}$ and ${ }_{2} D_{n}[U(z)]^{*}$ converge uniformly in $R$. Then

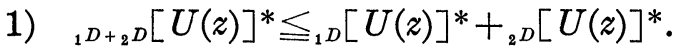

2) ${ }_{D}[C U(z)]^{*}=C_{D}[U(z)]^{*}$ for a constant $C$.

3) ${ }_{D}[U(z)]^{*} \leqq U_{D}(z) \leqq U(z)$.

4) Both ${ }_{D}[U(z)]^{*}$ and $U(z)-{ }_{D}[U(z)]^{*}$ are superharmonic in $\bar{R}$.

5) ${ }_{1} D[U(z)]^{*} \leqq_{2}[U(z)]$ for ${ }_{1} D \subset_{2} D$.

6) ${ }_{D}[U(z)]^{*}$ is representable by a mass distribution on $\bar{D} \cap B$, where $\bar{D}$ is the closure of $D$ with respect to Martin's topology.

7) ${ }_{B_{0}}[U(z)]^{*}=0$ for $U(z)=\int_{B_{0}} N(z, p) d \mu(p)$, where $B_{0}$ is the set of non-minimal points of $B$.

1), 2) and 5) are clear. Proof of 3). $D_{D}(z)=\lim _{n} U_{D_{n}}(z)$, where $U_{D_{n}}(z)$ is a harmonic function in $R-R_{0}-\left(D \cap R_{n}\right)$ such that $U(z)=U_{D_{n}}(z)$ on $D \cap R_{n}$ and $U_{D_{n}}(z)$ has M.D.I. over $R-R_{0}-\left(D \cap R_{n}\right)$. Now ${ }_{D_{n}}[U(z)]^{*}$ $\leqq U(z)=U_{D_{n}}(z)$ on $D \cap R_{n}$. On the other hand, since both $U_{D_{n}}(z)$ and ${ }_{D_{n}}[U(z)]^{*}$ have M.D.I. over $R-R_{0}-\left(D \cap R_{n}\right)$, by the maximum principle ${ }_{D_{n}}[U(z)]^{*} \leqq U_{D_{n}}(z)$. Hence

$$
{ }_{D}[U(z)]^{*}=\lim _{K=\infty}{ }_{D_{n^{\prime}}}[U(z)]^{*} \leqq \lim _{n} U_{D_{n}}(z)=U_{D}(z) .
$$

Proof of 4) and 6). ${ }_{D_{n}}[U(z)]^{*}$ and $U_{n}(z)-{ }_{D_{n}}[U(z)]^{*}$ are representable by positive mass distributions ${ }_{1} \mu_{n}$ and ${ }_{2} \mu_{n}\left(=\mu_{n}-{ }_{1} \mu_{n}\right)$ on $\partial R_{n} \cap \bar{D}$ and $\partial R_{n} \cap \overline{C D}$ respectively. But the total masses of ${ }_{1} \mu_{n}$ and ${ }_{2} \mu_{n}$ are bounded. We can find a subsequence $\left\{n^{\prime}\right\}$ of $\{n\}$ such that both $\left\{{ }_{1} \mu_{n^{\prime}}\right\}$ and $\left\{{ }_{2} \mu_{n^{\prime}}\right\}$ have weak limits ${ }_{1} \mu$ on $\bar{D} \cap \bar{R}$ and ${ }_{2} \mu$ on $\overline{C D} \cap \bar{R}$ respectively. Clearly by $\left\{n^{\prime}\right\} \subset\{n\}, \quad U(z)=\int N(z, p) d \mu(p), \quad{ }_{D}[U(z)]^{*}=\int N(z, p) d_{1} \mu(p)$ and $U(z)-{ }_{D}[U(z)]^{*}=\int N(z, p) d_{2} \mu(p)$. Hence ${ }_{D}[U(z)]^{*}$ and $U(z)-{ }_{D}[U(z)]^{*}$ are superharmonic in $\bar{R}$. Every $\mu_{n}$ is distributed on $\partial R_{n}$, whence ${ }_{1} \mu$ and ${ }_{2} \mu$ lie on $B$, whence ${ }_{D}[U(z)]^{*}$ and $U(z)-{ }_{D}[U(z)]^{*}$ are harmonic in $R$.

Proof of 7 ). We proved $U_{B_{0}}(z)^{2)}=0$. Hence by 3 ) we have 7).

3) of Theorem 8. Every positive harmonic function in $R-R_{0}$ such that $U(z)=0$ on $\partial R_{0}$ and $U(z)$ is superharmonic in $\bar{R}$ is representable by a positive mass distribution $\mu$ on $B_{1}$ such that

2) 2) of Theorem 8 . 


$$
U(z)=\int_{B_{1}} N(z, p) d \mu(p),
$$

where $B_{1}$ is the set of minimal points.

The set $\Gamma_{m}$ is defined as the set (possible void) of all points $q$ of such that $\psi\left(A_{m}(q)\right)=\frac{1}{2 \pi} \int_{\partial R_{0}} \frac{\partial N_{A_{m}(q)}(z, q)}{\partial n} d s \leqq \frac{1}{2} \quad($ this means $\psi(q)=0$ $\left.=\lim _{m} \psi\left(A_{m}(q)\right)\right)$, where $A_{m}(q)=E\left[z \in \bar{R}: \delta(z, q) \leqq \frac{1}{m}\right]$. Then $B_{0}=\bigcup_{m>0} \Gamma_{m}$. Suppose $U(z)=\int_{B_{0}} N(z, p) d \mu(p)$. Then by 2) of Theorem $8, U_{B_{0}}(z)=0$. And by 3) $U_{I_{m}^{\prime}}(z)=0$ implies $\lim _{n} \Gamma_{m, n}[U(z)]^{*}=0$, where $\Gamma_{m, n}=E[z \in \bar{R}$ : $\left.\delta\left(z, \Gamma_{m}\right) \leqq \frac{1}{n}\right]$. Let $z_{0}$ be a point in $R-R_{0}$. Then for any given positive number $\varepsilon$, there exists a number $n(m)$ such that

$$
\Gamma_{m, n}\left[U\left(z_{0}\right)\right]^{*} \leqq U_{\Gamma_{m, n}}\left(z_{0}\right) \leqq \frac{\varepsilon}{2^{2}} \quad \text { for } n \geqq n(m) .
$$

For each $m$ select $\Gamma_{m}^{\prime}\left(=\Gamma_{m, n}\right)$ in this fashion. Put $C_{m}=\sum_{i=1}^{m} \Gamma_{i}^{\prime}$. Then $C_{m}$ is closed and increases as $m \rightarrow \infty$. Denote by $\widetilde{A}_{m}$ and $A_{m}$ the closure of the complement of $C_{m}$ in $B$ and $\bar{R}$ respectively. Then the distance between $\widetilde{A}_{m}$ and $\Gamma_{m}$ is at least $\frac{1}{n(m)}$. Thus $\left\{\widetilde{A}_{n}\right\}$, which forms a decreasing sequence, has an intersection $\tilde{A}$ which is closed and, having no point in common with any $\Gamma_{m}$, is a subset $B_{1}$. Now

$$
{ }_{c_{m}}[U(z)]^{*} \leqq U_{C_{m}}(z) \leqq \sum^{m} U_{\Gamma_{i}^{\prime}}(z) \leqq \sum_{i}^{m} 2^{-i} \varepsilon \leqq \varepsilon \quad \text { for } z=z_{0}
$$

Observing $\widetilde{A}_{m}+\widetilde{C}_{m}=B$, we obtain for a subsequence $\left\{n^{\prime}\right\}$ of $\{n\}$ such that

$$
A_{m} \cap R_{n^{\prime}}[U(z)]^{*} \rightarrow \tilde{A}_{m}[U(z)]^{*} \quad \text { as } n^{\prime} \rightarrow \infty,
$$

where $A_{m} \cap B=\widetilde{A}_{m}$ and $A_{m}$ is a closed domain in $\bar{R}$.

$$
\tilde{A}_{m}[U(z)]^{*} \leqq \tilde{A}_{m}[U(z)]^{*}+c_{m}[U(z)]^{*} \leqq \tilde{A}_{m}[U(z)]^{*}+\varepsilon \geqq U(z)={ }_{B}[U(z)]^{*} \text {. }
$$

Now $U(z)-\tilde{A}_{m}[U(z)]^{*}$ and $\tilde{A}_{m}[U(z)]^{*}$ are representable by positive mass distributions $\mu_{m}^{\prime}$ and $\mu_{m}^{\prime \prime}$ over $C_{m} \cap B$ and $\widetilde{A}_{m}$ respectively. Let $\left\{n^{\prime \prime}\right\}$ be a subsequence of $\left\{n^{\prime}\right\}$ such that ${ }_{A_{m+1} \cap \bar{R}_{n^{\prime \prime}}}[U(z)]^{*} \rightarrow \widetilde{A}_{m+1}[U(z)]^{*}$. Then $\widetilde{A}_{m_{+1}}[U(z)]^{*}$ is representable by $\mu_{m+1}^{\prime \prime}$ over $\widetilde{A}_{m+1}$ and ${c_{m}}_{m}\left[U\left(z_{0}\right)\right]^{*}<\varepsilon$. Proceeding in this way, then by 5) $\tilde{A}_{m}[U(z)]^{*} \downarrow_{\tilde{A}}[U(z)]^{*}$ and ${c_{m}}_{m}\left[(U(z)]^{*}\right.$ $\uparrow_{\bar{c}}[U(z)]^{*}$, where $\bar{c}\left[U\left(z_{0}\right)\right]^{*}<\varepsilon$ by $c_{m}\left[U\left(z_{0}\right)\right]^{*}<\varepsilon$. $\mu_{m}^{\prime}$ and $\mu_{m}^{\prime \prime}$ have weak limits $\mu^{\prime}$ and $\mu^{\prime \prime}$ over $B \cap \bar{C}\left(=\overline{\sum C_{m}}\right)$ and $\widetilde{A}=\bigcap \widetilde{A}_{m} \subset B_{1}$ respectively. Hence

$$
U\left(z_{0}\right) \leqq \tilde{A}\left[U\left(z_{0}\right)\right]^{*}+\varepsilon,
$$

where $\tilde{A}[U(z)]^{*}$ and $U(z)-\tilde{A}[U(z)]^{*}$ are superharmonic in $\bar{R}$ and rep- 
resentable by ${ }_{1} \mu^{\prime}\left(=\mu^{\prime}\right)$ and ${ }_{1} \mu^{\prime \prime}\left(=\mu^{\prime \prime}\right)$. Let ${ }_{1} \mu^{\prime \prime \prime}$ be the restriction of ${ }_{1} \mu^{\prime \prime}$ on $B_{1}$ and put

$U_{1}(z)=\int_{B_{0}} N(z, p) d\left({ }_{1} \mu^{\prime \prime}-{ }_{1} \mu^{\prime \prime \prime}\right)$. Then $U_{1}(z) \leqq U(z)-\tilde{A}[U(z)]^{*}<\varepsilon$ for $z=z_{0}$ and

$$
U(z)-U_{1}(z)=\int_{B_{1}} N(z, p) d\left({ }_{1} \mu^{\prime}+{ }_{1} \mu^{\prime \prime \prime}\right)
$$

Repeat the process for $U_{1}(z)$, writting $U_{1}(z)=U_{2}(z)+\left(U_{1}(z)-U_{2}(z)\right)$, where $U_{2}(z)$ and $U_{1}(z)-U_{2}(z)$ are representable by mass distributions on $B_{0}$ and $B_{1}$ respectively and $U_{2}\left(z_{0}\right)<\frac{\varepsilon}{2}$.

Proceeding in this way $U_{n}(z)=U_{n+1}(z)-\left(U_{n}(z)-U_{n+1}(z)\right)$, where $U_{n+1}(z)$ and $U_{n}(z)-U_{n+1}(z)$ are representable by mass distributions over $B_{0}$ and $B_{1}$ respectively and $U_{n+1}\left(z_{0}\right)<\frac{\varepsilon}{2^{n}}$. Then

$$
U(z)=U(z)-U_{1}(z)+\sum_{n=1}^{\infty}\left(U_{n}(z)-U_{n+1}(z)\right)
$$

and $U(z)$ is represented by a mass distribution $\mu$ over $B_{1}$. Let $U(z)$ be a positive harmonic in $R$ and superharmonic in $\bar{R}$. Then $U(z)$ $=\int_{B} N(z, p) d \mu(p)$. Let $\mu^{\prime}$ be the restriction of $\mu$ over $B_{0}$. Then $\mu^{\prime}$ can be replaced by a distribution over $B_{1}$ and $\mu$ can be replaced by another distribution $\mu^{*}$ on $B_{1}$ without any change of $U(z)$. Hence we have the theorem.

Minimal point. Let $p$ be a point in $\bar{R}-R_{0}$ and let $F_{m}=E[z \in \bar{R}$ : $\left.\delta(z, \rho) \leqq \frac{1}{m}\right]$. If there exists a sequence ${ }_{F_{m}}[N(z, p)]^{*} \downarrow 0$ as $m \rightarrow \infty$, then we call $p$ a non-minimal point, otherwise a $\stackrel{*}{*}$ minimal point.

Theorem. Denote by $B_{1}^{*}$ and $B_{0}^{*}$ the set of minimal points and non-minimal points. Then

$$
B_{0}=B_{0}^{*} \quad \text { and } \quad B_{1}=B_{1}^{*} \text {. }
$$

Let $p \in B_{0}$. Then $N_{F_{m}}(z, p) \downarrow 0$ as $m \rightarrow \infty$ by Theorem 9 . Then by 3) ${ }_{F_{m}}[N(z, p)]^{*} \downarrow 0$, whence $p \in B_{0}^{*}$ and $B_{0} \subset B_{0}^{*}$.

Let $p \in B_{1}$. Then

$$
{ }_{B \cap F_{m}}[N(z, p)]^{*} \leqq N(z, p) \leqq{ }_{B \cap F_{m}}[N(z, p)]^{*}+_{B \cap \overline{C F}_{m}}[N(z, p)]^{*} \text {. }
$$

Now ${ }_{B \cap F_{m}}[N(z, p)]^{*},{ }_{B \cap \overline{C F}_{m}}[N(z, p)]^{*}, N(z, p)-{ }_{B \cap F_{m}}[N(z, p)]^{*}$ and $N(z, p)-{ }_{B \cap \overline{C F}_{m}}[N(z, p)]^{*}$ are superharmonic in $\bar{R}$. By the minimality of $N(z, p)_{B \cap F_{m}}[N(z, p)]=K N(z, p)$ and ${ }_{B \cap C F_{m}}[N(z, p)]=K^{\prime} N(z, p)$. Assume $K<1$, then $K^{\prime}>0$, which implies ${ }_{B \cap \overline{O F_{m}}}[N(z, p)]^{*}=K^{\prime} N(z, p)$ is represented by a positive mass distribution $\mu_{m}$ over $\overline{C F}_{m}$. Assume that $\mu_{m}$ is not a point mass, we can find a mass distribution $\mu^{\prime}$ such that $0<\mu_{n}^{\prime}<\mu_{m}$ and $\int N(z, p) d \mu_{n}^{\prime}(p)$ is not a multiple of $N(z, p)$, because 
if for every $\mu_{n}^{\prime}, \int N(z, p) d \mu_{n}^{\prime}(p)=K^{\prime} N(z, p)$, we can select a descending sequence of closed subsets $T_{n}$ in $\overline{C F}_{m}$ such that diameter of $T_{n} \rightarrow 0$ and $\cap T_{n}=q$. Put $\mu_{n}=\frac{\mu \text { on } T_{n}}{\text { total mass of } \mu}$ on $T_{n}$. Then $\left\{\mu_{n}\right\}$ has a weak limit mass at $q$ and $N(z, p)=N(z, q): q \in \overline{C F}_{m}$. This implies $p=q$. This is a contradiction. If $\mu_{m}$ is a point mass, then we have the same contradiction. Hence ${ }_{B \cap F_{m}}[N(z, p)]^{*}=N(z, p)$ for every $m$. Thus $p \in B_{1}^{*}$ and $B_{0}=B_{0}^{*}$ and $B_{1}=B_{1}^{*}$. 